\title{
Fully Developed Forced Convection in a Parallel Plate Channel with a Centered Porous Layer
}

\author{
Ozgur Cekmer • Moghtada Mobedi · Baris Ozerdem • \\ Ioan Pop
}

Received: 23 September 2011 / Accepted: 26 January 2012 / Published online: 15 February 2012

(C) Springer Science+Business Media B.V. 2012

\begin{abstract}
In this study, fully developed heat and fluid flow in a parallel plate channel partially filled with porous layer is analyzed both analytically and numerically. The porous layer is located at the center of the channel and uniform heat flux is applied at the walls. The heat and fluid flow equations for clear fluid and porous regions are separately solved. Continues shear stress and heat flux conditions at the interface are used to determine the interface velocity and temperature. The velocity and temperature profiles in the channel for different values of Darcy number, thermal conductivity ratio, and porous layer thickness are plotted and discussed. The values of Nusselt number and friction factor of a fully clear fluid channel $\left(N u_{\mathrm{cl}}=4.12\right.$ and $\left.f R e_{\mathrm{cl}}=24\right)$ are used to define heat transfer increment ratio $\left(\varepsilon_{\mathrm{th}}=N u_{\mathrm{p}} / N u_{\mathrm{cl}}\right)$ and pressure drop increment ratio $\left(\varepsilon_{\mathrm{p}}=f R e_{\mathrm{p}} / f R e_{\mathrm{cl}}\right)$ and observe the effects of an inserted porous layer on the increase of heat transfer and pressure drop. The heat transfer and pressure drop increment ratios are used to define an overall performance ( $\varepsilon=\varepsilon_{\mathrm{th}} / \varepsilon_{\mathrm{p}}$ ) to evaluate overall benefits of an inserted porous layer in a parallel plate channel. The obtained results showed that for a partially porous filled channel, the value of $\varepsilon$ is highly influenced from Darcy number, but it is not affected from thermal conductivity ratio $\left(k_{\mathrm{r}}\right)$ when $k_{\mathrm{r}}>2$. For a fully porous material filled channel, the value of $\varepsilon$ is considerably affected from thermal conductivity ratio as the porous medium is in contact with the channel walls.
\end{abstract}

Keywords Forced convection · Porous media - Heat transfer enhancement

O. Cekmer

Energy Engineering Program, Izmir Institute of Technology, 35430 Izmir, Turkey

M. Mobedi · B. Ozerdem

Department of Mechanical Engineering, Izmir Institute of Technology, 35430 Izmir, Turkey

B. Ozerdem

Department of Energy Systems Engineering, Bahcesehir University, 34353 Istanbul, Turkey

I. Pop (囚)

Faculty of Mathematics, University of Cluj, CP 253, 3400 Cluj, Romania

e-mail: popm.ioan@yahoo.co.uk 


\section{List of symbols}

\section{Variables}

$C_{p} \quad$ Specific heat at constant pressure $(\mathrm{J} / \mathrm{kg} \mathrm{K})$

$\mathrm{Da} \quad$ Darcy number

$G \quad$ Pressure gradient in $x$ direction

$h \quad$ Convective heat transfer coefficient $\left(\mathrm{W} / \mathrm{m}^{2} \mathrm{~K}\right)$

$H \quad$ Half height of channel (m)

$k \quad$ Thermal conductivity (W/m K)

$k_{\mathrm{f}} \quad$ Thermal conductivity of fluid (W/m K)

$k_{\text {eff }} \quad$ Effective thermal conductivity $(\mathrm{W} / \mathrm{m} \mathrm{K})$

$k_{\mathrm{r}} \quad$ Thermal conductivity ratio

$K \quad$ Permeability $\left(\mathrm{m}^{2}\right)$

$M \quad$ Dimensionless viscosity ratio

$\mathrm{Nu} \quad$ Nusselt number

$P \quad$ Pressure $(\mathrm{Pa})$

$q^{\prime \prime} \quad$ Constant heat flux subjected to the channel walls $\left(\mathrm{W} / \mathrm{m}^{2}\right)$

$S \quad$ Porous media shape parameter

$T \quad$ Temperature (K)

$T_{\mathrm{w}} \quad$ Wall temperature (K)

$u \quad$ Velocity component along the $x$ direction $(\mathrm{m} / \mathrm{s})$

$U$ Dimensionless velocity component along dimensionless $X$ direction

$u_{m} \quad$ Average velocity $(\mathrm{m} / \mathrm{s})$

$U_{m} \quad$ Dimensionless average velocity

$\hat{u} \quad$ Dimensionless normalized velocity

$x, y \quad$ Dimensional coordinates $(\mathrm{m})$

$X, Y$ Dimensionless coordinates

\section{Greek letters}

$\varepsilon \quad$ Overall performance

$\varepsilon_{\text {th }} \quad$ Heat transfer increment ratio

$\varepsilon_{\mathrm{p}} \quad$ Pressure drop increment ratio

$\xi \quad$ Dimensionless porous medium thickness

$\lambda \quad$ A constant (Eq. 17)

$\mu \quad$ Dynamic viscosity of fluid $(\mathrm{kg} / \mathrm{ms})$

$\mu_{\text {eff }}$ Effective dynamic viscosity $(\mathrm{kg} / \mathrm{ms})$

$\rho \quad$ Density $\left(\mathrm{kg} / \mathrm{m}^{3}\right)$

$\theta \quad$ Dimensionless temperature

$v \quad$ Kinematic viscosity of fluid $\left(\mathrm{m}^{2} / \mathrm{s}\right)$

\section{Introduction}

Convective heat transfer in porous media has broad technological practices, such as applications in oil recovery, water supply management in hydrogeology, geothermal exploitation, ground heat storage, building thermal insulation, nuclear waste disposals, radioactive waste management, and ground water flow modeling. A wide application areas of the porous media can be found in the well-known books by Bear (1972), Nield and Bejan (2006), Ingham and Pop (2005), Vafai (2005), Vafai (2010), Pop and Ingham (2001), Bejan et al. (2004), and 
Vadasz (2008). They provide significant information on the porous media application as well as formulation of heat and fluid flow in porous media.

Literature survey reveals that number of reported studies on channel partially filled with porous layer or porous blocks are considerable. However, the analytical studies on unidirectional fully developed heat and fluid flows in a channel partially filled with porous material are limited. Poulikakos and Kazmierczak (1987) investigated fully developed flow for a channel partially filled with porous matrix. The flow was fully developed both thermally and hydrodynamically. The matrix was attached to the walls and extended toward the centerline. The study was performed for two types of thermal boundary conditions as constant wall heat flux and constant wall temperature. Kuznetsov performed valuable studies on the forced convection heat transfer in a parallel plate channel partially filled with porous media. He studied heat and fluid flow in partially filled channels with different positions of porous layers such as porous layers attached to walls and located in the center of channel (Kuznetsov 1996, 1997, 1999, 2000). Brinkman-Forcheimer extended Darcy equation was analytically solved to determine velocity field, and the temperature field was found from the analytical solution of the ordinary differential equation form of the energy equation. Stress jump model was used to determine interface velocity while the model of continuous heat flux was employed for the calculation of the interface temperature. Kuznetsov (1998) also solved analytically a problem of heat transfer for Couette flow through a composite channel, which was partially filled with a fluid-saturated porous medium. The upper plate and the porous medium were fixed, while the lower plate was moving with a constant velocity. Kuznetsov and Nield (2010) investigated a fully developed forced convection in a parallel plate channel partially occupied by a bidisperse porous medium. One side of channel was filled by the clear fluid while bidisperse porous medium formed the other side. They found that for the case of asymmetric heating, a singular behavior of the Nusselt number exists. They also obtained analytical solution for fully developed forced convection in a channel between parallel plate walls, partly occupied by a bidisperse porous medium and partly by a fluid clear of solid material (Nield and Kuznetsov 2011). The bidisperse porous medium layer is either in the core or in the sheath portion of the channel. Beavers-Joseph interface condition was used in their study. Al-Nimr and Alkam (1998) studied, numerically, the thermal performance of a conventional tubeless collector in which porous substrates were inserted at the inner side of the collector absorber plate. The dimensionless form of Darcy-Brinkman-Forchheimer formulation was used to determine the motion of fluid in the porous layer. Satyamurty and Bhargavi (2010) performed a study on heat and fluid flow in a partially porous channel in which the inserted porous layer was attached to one wall while the other wall was in contact with clear fluid. Both walls were hold symmetrically at constant temperatures. The fluid flow in both regions was assumed to be fully developed, however, the temperature field was assumed under developing. Although an analytical solution was found for motion of fluid in the channel, the heat transfer equation was solved numerically. Yang et al. (2009) performed a theoretical study to investigate fully developed forced convection in a tube whose core was partially filled with a porous medium. Analytical expressions for the friction factor and Nusselt number for the case of constant heat flux were presented. In their study, Darcy law was used to simulate fluid motion in the porous layer. Chikh et al. (1995) performed an analytical study on fully developed forced convection in an annular duct partially filled with a porous medium. The porous layer was over the inner cylinder exposed to a constant heat flux. The outer surface was thermally insulated. Morosuk (2005) investigated entropy generation due to flow in a pipe and parallel plate channel partially filled with porous medium. The outer surface of the pipe was assumed to be in isothermal condition. The porous layer was inserted at the core of the pipe or attached on the inner surface of the pipe. The effects of the porous layer 
thickness and permeability of porous layer on the rate of entropy generation were investigated numerically. The effect of an inserted porous layer on enhancement of heat transfer in a parallel plate channel partially filled with a fluid-saturated porous medium was numerically studied by Shokouhmand et al. (2011). The fully developed laminar flow and convective heat transfer in the channel were simulated using Lattice Boltzmann Method (LBM). The walls of the channel were subject to a uniform constant temperature. It was found that the position of the inserted porous layer has significant influence on the thermal performance of the channel. Forooghi et al. (2011) studied, numerically, the steady and pulsatile heat and fluid flow in a channel with two porous layers subject to the constant wall heat flux under the local thermal non-equilibrium condition. The objective of the study was to assess the effects of local solid-to-fluid heat transfer and porous layer thickness on convective heat transfer and furthermore to examine the impact of pulsatile flow on heat transfer. The attention of some researchers who studied convection heat transfer in the channel partially filled with porous medium was focused on the discussion of boundary condition at the interface rather than analyzing of temperature and fluid flow for engineering applications (Vafai and Kim 1990; Alazmi and Vafai 2001; Nield 2009; Kuznetsov 1997). Further studies on heat and fluid flow analyses on channel and duct with different location of porous medium layer or obstacles can be found in the literature (Najjari and Nasrallah 2008; Ould-Amer et al. 1998; Alkam and Al-Nimr 1998).

The above literature survey reveals that some of reported studies were performed numerically and no mathematical expressions were presented for determination of velocity and temperature in the channel partially filled with porous medium. On the other hand, most of the reported analytical studies focused just on heat transfer analysis rather than the assessment of heat transfer enhancement and the increase of pressure drop due to an inserted porous layer into a channel. The studies on the evaluation of an inserted porous layer in a channel from the practical use point of view are very scarce (Al-Nimr and Alkam 1998). The objective of this study is to evaluate enhancement of heat transfer and increase of pressure drop in a channel due to an inserted porous layer when heat and fluid flows are fully developed. Furthermore, the comparison between heat transfer enhancement and increase of pressure drop is discussed in details. The study is performed for fully developed forced convection and mathematical expressions are obtained for the parameters of fluid velocity, temperature, Nusselt number, and friction coefficient. A constant heat flux is imposed at the channel walls whereas a thermal equilibrium established between the flowing fluid and porous medium. The Nusselt number and friction factor are calculated for channels with different values of Darcy number, porous layer thickness, and thermal conductivity ratios. Then, they are normalized by using Nusselt number and friction factor of a channel with completely clear fluid to find out the increasing of heat transfer and pressure drop due to the inserted porous layer. The normalized heat transfer and friction factor are called as heat transfer increment ratio and pressure drop increment ratio, respectively. These parameters present the effect of an inserted porous layer on heat transfer and pressure drop in the channel. The overall performance is defined to assess the benefits of an inserted porous medium layer in a channel. To the best of our knowledge, the results of this study make this article innovative one.

\section{Problem Definition}

A two-dimensional parallel plate channel whose core is filled with porous medium is studied in this article. The height of the channel is $2 H$ and the heat flux of $q^{\prime \prime}$ is imposed both at upper and lower walls as seen from Fig. 1. The porous layer with thickness of $2 \xi H$ is located at the 
Fig. 1 The schematic view of the considered channel

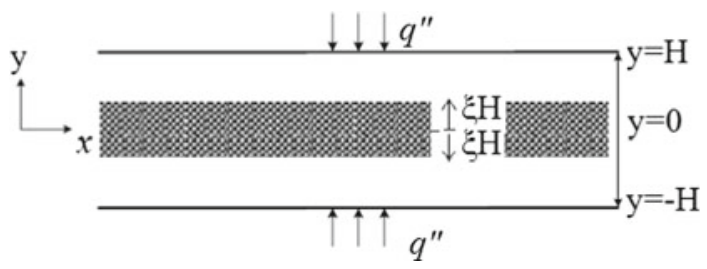

center of the channel. The Cartesian coordinates of $x$ and $y$ are axis in parallel and transverse directions of fluid flow, respectively. The porous medium is saturated with an incompressible fluid and local thermodynamic equilibrium state is valid. The flowing fluid in the channel is Newtonian whose thermophysical properties are assumed as constant. The porous medium is isotropic with permeability of $K$. The flow in the channel is steady, laminar, and hydrodynamically and thermally fully developed. Table 1 is prepared to determine numeral ranges for Darcy number. In Table 1, the values of Darcy number for two porous layer configurations as a square bar and a metal foam located in the center of channel with $30-\mathrm{mm}$ height are calculated. For the square bar porous layer configuration, the center of channel is filled with square bars, and fluid flows perpendicularly over the bars. For square bar porous layer, the value of permeability is calculated by using relation proposed by Nakayama et al. (2007). However, for metal foam, the value of permeability is taken from the study of Calmidi and Mahajan (2000). The value of Darcy number for each configuration of porous layer is calculated. As seen, the value of Darcy number can be changed between $10^{-2}$ and $10^{-5}$. The value of dimensionless viscosity ratio is accepted as 1.1 for this study. The range of effective thermal conductivity can be changed from 0.1 to $10^{4}$. For instance, the effective thermal conductivity of air-rigid rubber for $\varphi=0.35$ is 1.14 (i.e., $k_{\text {eff }}=1.14$ ), while for air-Al (pure) and the same porosity value $k_{\text {eff }}=5,858$.

\section{Governing Equations and Boundary Conditions}

\subsection{Fluid Flow}

The flow in the channel is hydrodynamically fully developed and unidirectional in $x$ direction. Considering Fig. 1, no gradient of $u$ velocity component in flow direction exits, hence $\partial u / \partial x=0$. The momentum equation for the clear portion of the channel can be written as follows:

$$
v \frac{\partial^{2} u_{\mathrm{c}}}{\partial y^{2}}-\frac{1}{\rho} \frac{\partial P}{\partial x}=0,
$$

where $u_{\mathrm{c}}$ represents fluid velocity in the clear region of the channel (the subscript "c" stands for clear portion of the channel), $v$ is kinematic viscosity, $\rho$ is density, and $P$ is pressure. The motion equation for the porous part of the channel can be written as:

$$
\mu_{\text {eff }} \frac{\mathrm{d}^{2} u_{\mathrm{p}}}{\mathrm{d} y^{2}}-\frac{\mu}{K} u_{\mathrm{p}}-\frac{\partial P}{\partial x}-\frac{C_{\mathrm{f}} \rho u_{\mathrm{p}}}{\sqrt{K}}=0,
$$

where $\mu$ is dynamic viscosity of the fluid, $\mu_{\text {eff }}$ is effective dynamic viscosity of the fluid and porous medium, $u_{\mathrm{p}}$ shows fluid velocity in porous layer (the subscript " $\mathrm{p}$ " stands for porous portion in the channel), $K$ and $C_{\mathrm{f}}$ are permeability and inertial coefficient, respectively. The first term in Eq. 2 is written to involve the channel wall effect on the fluid motion. The second 
and third terms are related to Darcy law and the fourth term, called as Forchheimer term, involves the inertia effect. As seen from Table 1, for some engineering application, the value of Reynolds number based on the particle size is small. This indicates that the Forchheimer term can be neglected in some practical porous media applications. In this study, the Forcheimer term is not considered. Boundary conditions for the Eqs. 1 and 2 can be written as:

$$
u_{\mathrm{c}}( \pm H)=0, \quad u_{\mathrm{c}}( \pm \xi H)=u_{\mathrm{p}}( \pm \xi H), \quad \tau_{\mathrm{c}}( \pm \xi H)=\tau_{\mathrm{p}}( \pm \xi H)
$$

where $\tau_{\mathrm{c}}$ and $\tau_{\mathrm{p}}$ show shear stress at the interface surface. One of the hottest topics in porous media is the determination of interface velocity at porous medium and clear fluid regions. Many studies and comments on the interface velocity were performed (e.g., Kuznetsov 1997; Nield 2009; Vafai and Kim 1990). Nevertheless, the controversy on thermal and hydrodynamic model for the interface continues. In this study, to circumvent the controversy, and basically to focus on the enhancement of heat transfer and increase of pressure drop, the continuous shear stress model is used to define the boundary condition for fluid motion at the interface between porous layer and clear fluid regions. Although the channel is symmetrical respect to the center line, the governing equations are solved for three different regions as lower clear fluid region, center porous layer, and upper clear fluid regions. By this way, the correctness of obtained result is checked as the temperature and velocity fields should be symmetrical to the centerline. To reduce the number of the parameters for the problem, the dimensionless forms of Eqs. 1 and 2 and related boundary conditions (Eq. 3) are obtained by using the following dimensionless parameters:

$$
Y=\frac{y}{H}, M=\frac{\mu_{\mathrm{eff}}}{\mu}, D a=\frac{K}{H^{2}}, G=-\frac{\partial P}{\partial x}, S=\frac{1}{\sqrt{M D a}}, \theta=\frac{T-T_{\mathrm{w}}}{T_{\mathrm{m}}-T_{\mathrm{w}}}, U=\frac{\mu u}{G H^{2}},
$$

where $M$ and $S$ are viscosity ratio and porous media shape parameter, respectively. After substituting of the above dimensionless parameters into Eqs. 1 and 2, the dimensionless forms of the momentum equation for fluid flow in the clear regions and Darcy-Brinkman equation for the fluid flow in the porous region can be written as:

$$
\begin{aligned}
& \frac{\mathrm{d}^{2} U_{\mathrm{c}}}{\mathrm{d} Y^{2}}+1=0 \\
& \frac{\mathrm{d}^{2} U_{\mathrm{p}}}{\mathrm{d} Y^{2}}-S^{2} U_{\mathrm{p}}+\frac{1}{M}=0 .
\end{aligned}
$$

$U_{\mathrm{c}}$ and $U_{\mathrm{p}}$ are dimensionless velocity of fluid in the clear and porous regions. The dimensionless forms of boundary conditions can also be defined by using the dimensionless parameters of Eq. 4.

$$
U_{\mathrm{c}}( \pm 1)=0, \quad U_{\mathrm{c}}( \pm \xi)=U_{\mathrm{p}}( \pm \xi), \quad \tau_{\mathrm{c}}( \pm \xi)=\tau_{\mathrm{p}}( \pm \xi)
$$

The solutions of Eqs. 5 and 6 with boundary conditions given in Eq. 7 yield the velocity profile equations for each region;

$$
\begin{aligned}
& \text { For }-1 \leq Y<-\xi \text { region } U(Y)=A_{1} Y^{2}+A_{2} Y+A_{3} \\
& \text { For }-\xi \leq Y \leq \xi \text { region; } U(Y)=P_{1} \cosh (S Y)+P_{2} \\
& \text { For } \xi<Y \leq 1 \text { region } U(Y)=A_{1} Y^{2}+A_{4} Y+A_{3},
\end{aligned}
$$




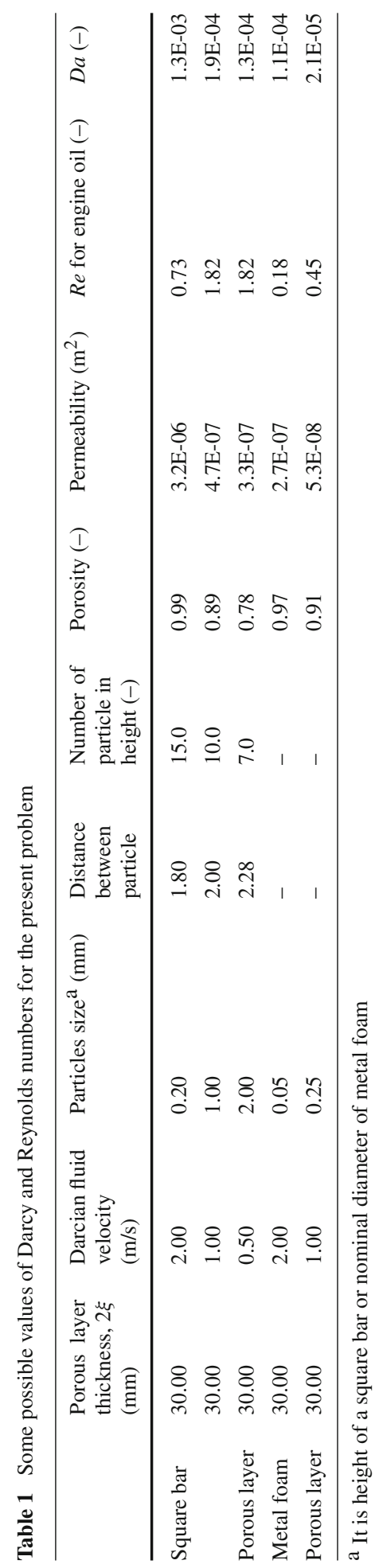


where $A_{1}, A_{2}, A_{3}, A_{4}, P_{1}$, and $P_{2}$ are constants given by

$$
\begin{aligned}
& A_{1}=-\frac{1}{2}, \quad A_{2}=\frac{\xi^{2}+2 V-1}{2(\xi-1)}, \quad A_{3}=\frac{\xi-\xi^{2}-2 V}{2(\xi-1)}, \quad A_{4}=\frac{1-2 V-\xi^{2}}{2(\xi-1)} \\
& P_{1}=\frac{\left(V S^{2} M-1\right) \operatorname{sech}(S \xi)}{M S^{2}}, \quad P_{2}=\frac{1}{M S^{2}} .
\end{aligned}
$$

Here, $V$ is the dimensionless velocity at the interface between the porous and clear region [i.e., $\left.V=U_{\mathrm{c}}( \pm \xi)=U_{\mathrm{p}}( \pm \xi)\right]$. The shear stress is continuous at the surface $\left[\tau_{\mathrm{c}}( \pm \xi)=\tau_{\mathrm{p}}( \pm \xi)\right]$, therefore the interface velocity can be found as:

$$
V=\frac{[2 \tanh (S \xi)-S \xi+S](\xi-1)}{2 S[(\xi-1) \mathrm{MS} \tanh (S \xi)-1]} .
$$

The mean velocity can be defined as:

$$
U_{\mathrm{m}}=\frac{\int_{-1}^{1} U \mathrm{~d} Y}{2}=\frac{\int_{-1}^{-\xi} U_{\mathrm{c}}(Y) \mathrm{d} Y+\int_{-\xi}^{+\xi} U_{\mathrm{p}}(Y) \mathrm{d} Y+\int_{+\xi}^{1} U_{\mathrm{c}}(Y) \mathrm{d} Y}{2} .
$$

By substituting Eqs. 8-10 into Eq. 13 and taking integral, the mean velocity is obtained as:

$$
U_{\mathrm{m}}=\frac{A_{1}}{3}\left(1-\xi^{3}\right)+\frac{A_{2}-A_{4}}{4}\left(\xi^{2}-1\right)+A_{3}(1-\xi)+\frac{P_{1}}{S} \sinh (S \xi)+P_{2} \xi .
$$

The constants $A_{1}, A_{2}, A_{3}, A_{4}, P_{1}$, and $P_{4}$ are presented in Eq. 11. The dimensionless normalized velocity $\hat{u}$ can be determined as:

$$
\hat{u}=\frac{u}{u_{\mathrm{m}}}=\frac{U}{U_{\mathrm{m}}} .
$$

\subsection{Heat Flow}

For a thermally and hydrodynamically fully developed laminar flow in a partially porous filled parallel plate channel, the energy equation can be written as:

$$
\left(\rho C_{p}\right)_{f} u \frac{\partial T}{\partial x}=\left[\lambda\left(k_{\mathrm{eff}}-k_{\mathrm{f}}\right)+k_{\mathrm{f}}\right] \frac{\partial^{2} T}{\partial y^{2}},
$$

where $k_{\mathrm{f}}$ and $k_{\text {eff }}$ are thermal conductivity of the fluid and effective conductivity of the porous structure, respectively. The parameter of $\lambda$ depends on the region and it is defined as:

$$
\begin{array}{r}
-1 \leq Y<-\xi=>\lambda=0 \\
-\xi \leq Y \leq \xi=>\lambda=1 \\
\xi<Y \leq 1=>\lambda=0 .
\end{array}
$$

As the heat fluxes applied to both walls are held constant and equal, and the porous layer is placed in the center of channel symmetrically, the wall temperatures are identical at both upper and inner walls and designated by $T_{\mathrm{w}}$. The energy equation can be written in the dimensionless form by using the dimensionless parameters defined in Eq. 4. After some mathematical manipulations, the energy equation takes the following form,

$$
\rho C_{p} u\left[\theta\left(\frac{\partial T_{\mathrm{m}}}{\partial x}-\frac{\partial T_{\mathrm{w}}}{\partial x}\right)+\left(T_{\mathrm{m}}-T_{\mathrm{w}}\right) \frac{\partial \theta}{\partial x}+\frac{\partial T_{\mathrm{w}}}{\partial x}\right]=\left[\lambda\left(k_{\mathrm{eff}}-k_{\mathrm{f}}\right)+k_{\mathrm{f}}\right]\left(T_{\mathrm{m}}-T_{\mathrm{w}}\right) \frac{\partial^{2} \theta}{\partial y^{2}} .
$$


As stated above, the applied heat fluxes at both walls are held constant. Therefore, the gradient of the heat fluxes along the channel is zero:

$$
\frac{\mathrm{d} q^{\prime \prime}}{\mathrm{d} x}=\frac{\mathrm{d}\left[h\left(T_{\mathrm{w}}-T_{\mathrm{m}}\right)\right]}{\mathrm{d} x}=0
$$

and therefore, we reach to

$$
\frac{\mathrm{d} T_{\mathrm{w}}}{\mathrm{d} x}=\frac{\mathrm{d} T_{m}}{\mathrm{~d} x} .
$$

The substitution of Eq. 19 into Eq. 18 and using the thermally fully developed condition $(\partial \theta / \partial x=0)$, the energy equation takes the following form:

$$
\rho C_{p} u \frac{\mathrm{d} T_{\mathrm{m}}}{\mathrm{d} x}=\left[\lambda\left(k_{\mathrm{eff}}-k_{\mathrm{f}}\right)+k_{\mathrm{f}}\right]\left(T_{\mathrm{m}}-T_{\mathrm{w} \mu}\right) \frac{\mathrm{d}^{2} \theta}{\mathrm{d} y^{2}} .
$$

To find a definition for the mean temperature gradient in $x$ direction $\left(\mathrm{d} T_{\mathrm{m}} / \mathrm{d} x\right)$, an energy balance is applied for a control volume in the cross section of the channel and the following result can be found:

$$
\frac{\mathrm{d} T_{\mathrm{m}}}{\mathrm{d} x}=\frac{q^{\prime \prime}}{\rho C_{p} u_{\mathrm{m}} H} .
$$

By substituting Eq. 22 into Eq. 21, the following equation is obtained.

$$
\frac{u}{u_{\mathrm{m}}} \frac{q^{\prime \prime}}{H}=\left[\lambda\left(k_{\mathrm{eff}}-k_{\mathrm{f}}\right)+k_{\mathrm{f}}\right]\left(T_{\mathrm{m}}-T_{\mathrm{w}}\right) \frac{\mathrm{d}^{2} \theta}{\mathrm{d} y^{2}} .
$$

The heat flux to the fluid can be expressed as:

$$
q^{\prime \prime}=h\left(T_{\mathrm{w}}-T_{\mathrm{m}}\right) \text {. }
$$

Replacing Eq. 24 into Eq. 23 yields the following second order ordinary differential equation:

$$
\frac{\mathrm{d}^{2} \theta}{\mathrm{d} Y^{2}}+\hat{u} \frac{h H}{\left[\lambda\left(k_{\mathrm{eff}}-k_{\mathrm{f}}\right)+k_{\mathrm{f}}\right]}=0 .
$$

The local Nusselt number can be defined as:

$$
N u=\frac{(2 H) h}{k_{\mathrm{f}}} .
$$

Substituting definition of Nusselt number into Eq. 25 yields the heat transfer equation for entire domain of fully developed channel.

$$
\frac{\mathrm{d}^{2} \theta}{\mathrm{d} Y^{2}}+\frac{1}{2} \hat{u} N u \frac{1}{\left[\lambda\left(k_{\mathrm{r}}-1\right)+1\right]}=0,
$$

where $k_{\mathrm{r}}$ is thermal conductivity ratio and defined as:

$$
k_{\mathrm{r}}=\frac{k_{\mathrm{eff}}}{k_{\mathrm{f}}} \text {. }
$$

The energy equation for the porous layer and clear regions can be written as:

$$
\begin{aligned}
& \frac{\mathrm{d}^{2} \theta}{\mathrm{d} Y^{2}}+\frac{1}{2 k_{\mathrm{r}}} \hat{u} N u=0 \text { for porous layer region } \\
& \frac{\mathrm{d}^{2} \theta}{\mathrm{d} Y^{2}}+\frac{1}{2} \hat{u} N u=0 \text { for upper and lower clear regions. }
\end{aligned}
$$


Assuming non-slip temperature at the interface and continuous heat flux through the porous layer and clear regions, the boundary conditions for Eq. 29 can be written as:

$$
\begin{array}{lll}
T_{\mathrm{c}}(H)=T_{\mathrm{w}} & T_{\mathrm{c}}(H \xi)=T_{\mathrm{p}}(H \xi) & \left.k_{f} \frac{\mathrm{d} T_{\mathrm{c}}}{\mathrm{d} y}\right|_{y=H \xi}=\left.k_{\mathrm{eff}} \frac{\mathrm{d} T_{\mathrm{p}}}{\mathrm{d} y}\right|_{y=H \xi} \\
T_{\mathrm{c}}(-H)=T_{\mathrm{w}} & T_{\mathrm{c}}(-H \xi)=T_{\mathrm{p}}(-H \xi) & \left.k_{f} \frac{\mathrm{d} T_{\mathrm{c}}}{\mathrm{d} y}\right|_{y=-H \xi}=\left.k_{\mathrm{eff}} \frac{\mathrm{d} T_{\mathrm{p}}}{\mathrm{d} y}\right|_{y=-H \xi}
\end{array}
$$

Using the dimensionless parameters defined by Eq. 4 yields dimensionless form of the boundary conditions:

$$
\begin{array}{lll}
\theta_{\mathrm{c}}(1)=0 & \theta_{\mathrm{c}}(\xi)=\theta_{\mathrm{p}}(\xi) & \left.\frac{\mathrm{d} \theta_{\mathrm{c}}}{\mathrm{d} Y}\right|_{Y=\xi}=\left.k_{\mathrm{r}} \frac{\mathrm{d} \theta_{\mathrm{p}}}{\mathrm{d} Y}\right|_{Y=\xi} \\
\theta_{\mathrm{c}}(-1)=0 & \theta_{\mathrm{c}}(-\xi)=\theta_{\mathrm{p}}(-\xi) & \left.\frac{\mathrm{d} \theta_{\mathrm{c}}}{\mathrm{d} Y}\right|_{Y=-\xi}=\left.k_{\mathrm{r}} \frac{\mathrm{d} \theta_{\mathrm{p}}}{\mathrm{d} Y}\right|_{Y=-\xi}
\end{array}
$$

The solution of the second order ordinary differential equation of (27) with the boundary conditions of (31) yields temperature distribution along the height of the channel.

$$
\begin{array}{rll}
\text { For }-1 \leq Y<-\xi \text { region: } & \theta(Y)=C_{6}+C_{7} Y+C_{3} Y^{2}+C_{8} Y^{3}+C_{5} Y^{4} \\
\text { For }-\xi \leq Y \leq \xi \text { region: } & \theta(Y)=C_{9}+C_{10} Y^{2}+C_{11} \cosh (S Y) \\
\text { For } \xi<Y \leq 1 \text { region: } & \theta(Y)=C_{1}+C_{2} Y+C_{3} Y^{2}+C_{4} Y^{3}+C_{5} Y^{4},
\end{array}
$$

where coefficients from $\mathrm{C}_{1}$ to $\mathrm{C}_{11}$ are given by

$$
\begin{aligned}
C_{1} & =\frac{24 U_{m} \theta_{\mathrm{i}}+N u \xi(\xi-1)\left[2\left(A_{2}(\xi+1)+3 A_{3}\right)+A_{1}\left(1+\xi+\xi^{2}\right)\right]}{24 U_{m}(1-\xi)} \\
C_{2} & =\frac{24 U_{m} \theta_{\mathrm{i}}+N u\left[6 A_{3}\left(\xi^{2}-1\right)+2 A_{2}\left(\xi^{3}-1\right)+A_{1}\left(\xi^{4}-1\right)\right]}{24 U_{m}(\xi-1)} \\
C_{3} & =-\frac{A_{3} N u}{4 U_{m}}, \quad C_{4}=-\frac{A_{2} N u}{12 U_{m}}, \quad C_{5}=-\frac{A_{1} N u}{24 U_{m}} \\
C_{6} & =\frac{-24 U_{m} \theta_{\mathrm{i}}+N u \xi\left[A_{1}\left(1-\xi^{3}\right)-6 A_{3}(\xi-1)+2 A_{4}\left(\xi^{2}-1\right)\right]}{24 U_{m}(\xi-1)} \\
C_{7} & =\frac{24 U_{m} \theta_{\mathrm{i}}+N u\left[A_{1}\left(\xi^{4}-1\right)-2 A_{4}\left(\xi^{3}-1\right)+6 A_{3}\left(\xi^{2}-1\right)\right]}{24 U_{m}(1-\xi)} \\
C_{8} & =-\frac{A_{4} N u}{12 U_{m}}, \quad C_{9}=\frac{4 S^{2} k_{\mathrm{r}} U_{m} \theta_{\mathrm{i}}+N u\left[S^{2} P_{2} \xi^{2}+2 P_{1} \cosh (S \xi)\right]}{4 S^{2} k_{\mathrm{r}} U_{m}} \\
C_{10} & =-\frac{N u P_{2}}{4 k_{\mathrm{r}} U_{m}}, \quad C_{11}=-\frac{N u P_{1}}{2 k_{\mathrm{r}} U_{m} S^{2}} .
\end{aligned}
$$

In the above relations, the term $\theta_{\mathrm{i}}$ designates the dimensionless interface temperature of porous and clear regions. The interface temperature between porous and clear fluid can be found by using condition of continues heat flux at interface surface. After performing some mathematical manipulations, the temperature at the interface, $\theta_{\mathrm{i}}$ can be obtained as:

$$
\theta_{\mathrm{i}}=\psi N u
$$


where coefficient of $\psi$ is defined as:

$$
\begin{gathered}
\psi=\sum_{k=1}^{3} \psi_{k} \\
\psi_{1}=\frac{(\xi-1)\left[P_{2} S-P_{1} \sinh (S \xi)\right]}{2 U_{m} S}, \quad \psi_{2}=\frac{6 A_{3}\left(1-\xi^{2}\right)+2 A_{2}\left(1-\xi^{3}\right)+A_{1}\left(1-\xi^{4}\right)}{24 U_{m}} \\
\psi_{3}=\frac{(1-\xi)}{2 U_{m}}\left[A_{3} \xi+\frac{A_{2} \xi^{2}}{2}+\frac{A_{1} \xi^{3}}{3}\right] .
\end{gathered}
$$

To find the Nusselt number, the compatibility condition (Eq. 37) can be applied.

$$
\int_{-1}^{1} \hat{u} \theta \mathrm{d} Y=2 .
$$

After applying the compatibility condition, the following equation for Nusselt number is obtained

$$
N u=\frac{2 U_{m}}{\sum_{k=1}^{5} N_{k}},
$$

where the terms from $N_{1}$ to $N_{5}$ are constants and they are found as:

$$
\begin{aligned}
& N_{1}=\frac{\psi(1-\xi)\left[8 V+(\xi-1)^{2}\right]}{12}, \quad N_{2}=\frac{(1-\xi)^{3}\left[448 V^{2}+168 V(\xi-1)^{2}+17(\xi-1)^{4}\right]}{20160 U_{m}} \\
& N_{3}=\frac{\operatorname{sech}^{2}(S \xi) \xi\left(\left[\xi^{2}-3 M V\left(M S^{2} V-4\right)\right] S^{2}-9+6 k_{\mathrm{r}} M \psi S^{4} U_{m}\right)}{6 k_{\mathrm{r}} M^{2} S^{6} U_{m}} \\
& N_{4}=\frac{\operatorname{sech}^{2}(S \xi) \cosh (2 S \xi) \xi^{2}\left(S^{2}\left[6 M\left(k_{\mathrm{r}} \psi S^{2} U_{m}+V\right)+\xi^{2}\right]-6\right)}{3 k_{\mathrm{r}} M^{2} S^{5} U_{m}} \\
& N_{5}=\frac{\operatorname{sech}^{2}(S \xi) \sinh (2 S \xi) \xi\left(M S^{2} V-1\right)\left(M S^{2} V-5+4 k_{\mathrm{r}} M \psi S^{4} U_{m}\right)}{2 k_{\mathrm{r}} M^{2} S^{6} U_{m}} .
\end{aligned}
$$

\subsection{Pressure Drop}

The use of porous media in a channel increases heat transfer through the channel, however, it increases pressure drop through the channel, as well. That is why, both heat transfer and pressure drop should be analyzed in heat transfer enhancement problems. Friction factor for a channel in which fluid flows with $u_{m}$ is defined as:

$$
f=\frac{(2 H)\left(-\frac{\mathrm{d} P}{\mathrm{~d} x}\right)}{\frac{1}{2} \rho u_{m}^{2}} .
$$

For a fully developed flow in a channel, the pressure gradient along the $x$ axis is constant and this negative gradient is denoted by $G$ in this study. Then Eq. 40 becomes as follows:

$$
f=\frac{4 G H}{\rho u_{m}^{2}} .
$$


By using the definition of the dimensionless velocity defined in Eq. 4, the following equation is obtained for the friction factor.

$$
f R e=\frac{8}{U_{m}} .
$$

Equation 42 is a measure of the pressure drop and it can be used to compare pressure drop in the channels with different Darcy numbers and porous layer thicknesses.

\subsection{Analysis of Heat Transfer and Pressure Drop Increments}

To determine the dimensionless heat transfer from a wall to a fluid, the Nusselt number is defined (Eq. 26). However, it might be useful to normalize the Nusselt number by using the Nusselt number of a fully clear fluid channel to understand the effect of porous layer on heat transfer. The normalized Nusselt number is called as heat transfer increment ratio in this study.

$$
\varepsilon_{\mathrm{th}}=\frac{N u}{N u_{\mathrm{c}}} .
$$

For a clear fluid channel, the value of heat transfer increment ratio is unity, $\varepsilon_{\text {th }}=1$. Heat transfer enhancement is expected for the channel with $\varepsilon_{\mathrm{th}}>1$. The use of porous layer may decrease heat transfer through a channel if $\varepsilon_{\text {th }}<1$. For this problem, the value of Nusselt number for a fully clear fluid channel is found as 4.12 (i.e., $N u_{\mathrm{c}}=4.12$ ). Similarly, the pressure drop increment ratio can be defined by using the friction coefficient (Eq. 42). The friction coefficient can be normalized based on the friction coefficient of a channel with pure fluid.

$$
\varepsilon_{\mathrm{P}}=\frac{(f R e)_{\mathrm{p}}}{(f R e)_{\mathrm{c}}} .
$$

For the values of pressure drop increment ratio greater than 1, the increase of pressure drop in the channel is expected. The value of friction coefficient for a fully clear fluid channel $(f R e)_{\mathrm{cl}}$ is found as 24 [i.e., $(f R e)_{\mathrm{cl}}=24$ ].

The ratio of $\varepsilon_{\mathrm{p}}$ and $\varepsilon_{\mathrm{th}}$ is called as overall performance and shown with $\varepsilon$. The overall performance compares the heat transfer enhancement and pressure drop by inserting a porous layer in a channel.

$$
\varepsilon=\frac{\varepsilon_{\mathrm{th}}}{\varepsilon_{\mathrm{p}}} .
$$

For the values of $\varepsilon$ greater than 1 (i.e., $\varepsilon>1$ ), the heat transfer enhancement is greater than the increase of pressure drop if a porous layer is used at the core of the channel. However, for the values of $\varepsilon$ less than 1 (i.e., $\varepsilon<1$ ), the increase of pressure drop in the channel is greater than heat transfer enhancement.

For this study, the channel walls are only in contact with fluid, and Nusselt number is defined based on fluid thermal conductivity. However, results for the channels completely filled with fully porous materials are also evaluated. For a channel with fully porous media, Nusselt number is generally defined based on the effective thermal conductivity as the channel walls are in contact with both fluid and porous material. In this study, to compare the Nusselt numbers of the partially and fully filled porous channel with each others, Nusselt number of channel filled with fully porous material is defined based on fluid thermal conductivity:

$$
N u_{\mathrm{fp}}=N u_{\mathrm{p}} k_{\mathrm{r}},
$$


where $N u_{\mathrm{fp}}$ refers to Nusselt number of channel filled with fully porous material based on fluid thermal conductivity. and $N u_{\mathrm{p}}$ is Nusselt number based on the effective thermal conductivity.

\section{Numerical Solution Procedure}

In this study, the velocity and temperature fields are also obtained, numerically, for both clear fluid and porous regions. Based on the obtained temperature distribution, Nusselt number is numerically calculated, as well. Finite difference method is used to convert the ordinary differential equations to the set of linear algebraic equations solved by Gauss Sidel iteration method. The velocity field can be found from Eqs. 5 and 6 under the boundary condition of Eq. 7, easily. The value of velocity at the interface is numerically determined from continuous shear stress condition. For the energy equations, the following transformation is used. The function of $\Psi$ is defined such that

$$
\Psi=\frac{\theta}{N u} .
$$

The overall Nusselt number is constant for fully developed flow in a channel. Hence, a new form of dimensionless heat transfer equation and boundary conditions based on $\psi$ function can be written.

$$
\frac{\mathrm{d}^{2} \Psi}{\mathrm{d} Y^{2}}+\frac{1}{2} \hat{u}=0
$$

and

$$
\Psi(-1)=0, \quad \Psi(1)=0 .
$$

The continuous heat flux at interface is employed to determine value of $\psi$ at the interface. The new form of the compatibility condition is:

$$
\int_{-1}^{1} \Psi \hat{u} \mathrm{~d} Y=2 / N u .
$$

Equation 48 under boundary condition of (49) is numerically solved to determine distribution of $\Psi$ for the channel. Then, using Eq. 50, the value of Nusselt number is obtained.

\section{Result and Discussion}

Based on the obtained analytical results, the velocity and temperature profiles for different Darcy number, thermal conductivity ratio, and porous layer thickness are plotted. The variations of heat transfer and pressure drop increment ratios and overall performance against the governing parameters are presented via graphics for the ranges of $10^{-5}<\mathrm{Da}<10^{-2}, 0.1<$ $k_{\mathrm{r}}<100$ and $0<\xi<1$. As mentioned above, the results of analytical expressions for the velocity, temperature, and Nusselt number are compared with the obtained numerical results. Figure 2 compares the temperature profiles obtained by analytical and numerical methods for a channel with $D a=10^{-3}, M=1.1$, and $\xi=0.6$ and for two values of $k_{\mathrm{r}}$ as 0.1 and 100 . As seen from the figure, there is a good agreement between numerical and analytical results showing correctness of the obtained analytical expression. Furthermore, the obtained results 
Fig. 2 The comparison of analytical and numerical results by temperature profile for two values of $k_{\mathrm{r}}$ when $D a=10^{-3}$, $M=1.1$, and $\xi=0.6$

Fig. 3 Comparison of present and reported studies results a velocity profile for $M=1$ and $D a=10^{-4}$, b Nusselt number for $M=1$ and $k_{\mathrm{r}}=1$
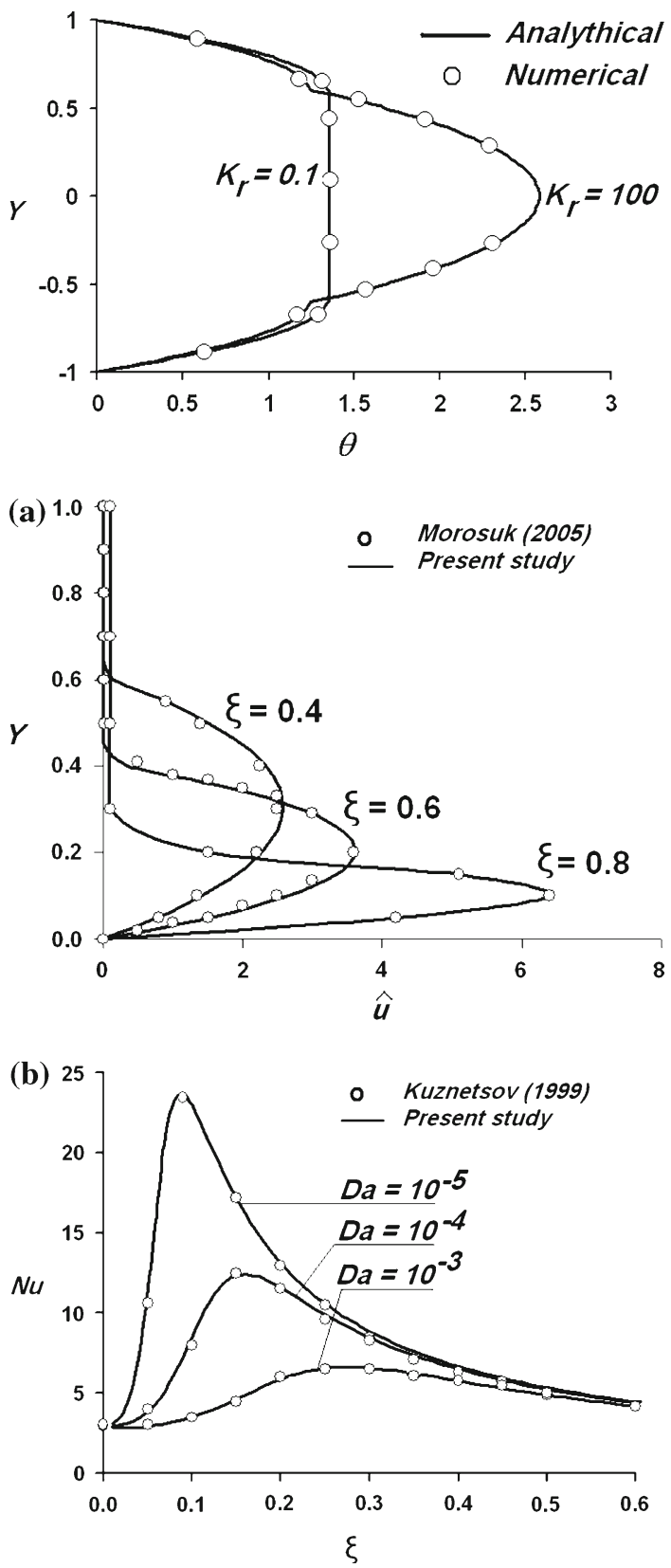

of this study are also checked with the results reported in the literature. Figure 3 a shows the comparison of the velocity profile obtained in this study with the profile reported by Morosuk (2005) when $M=1, D a=10^{-4}$. Figure $3 \mathrm{~b}$ indicates the comparison of changes in Nusselt with Darcy number of this study and the study performed by Kuznetsov (1999) for a channel with $M=1$ and $k_{\mathrm{r}}=1$. As seen from both the figures, a good agreement between the present and reported studies can be observed. 

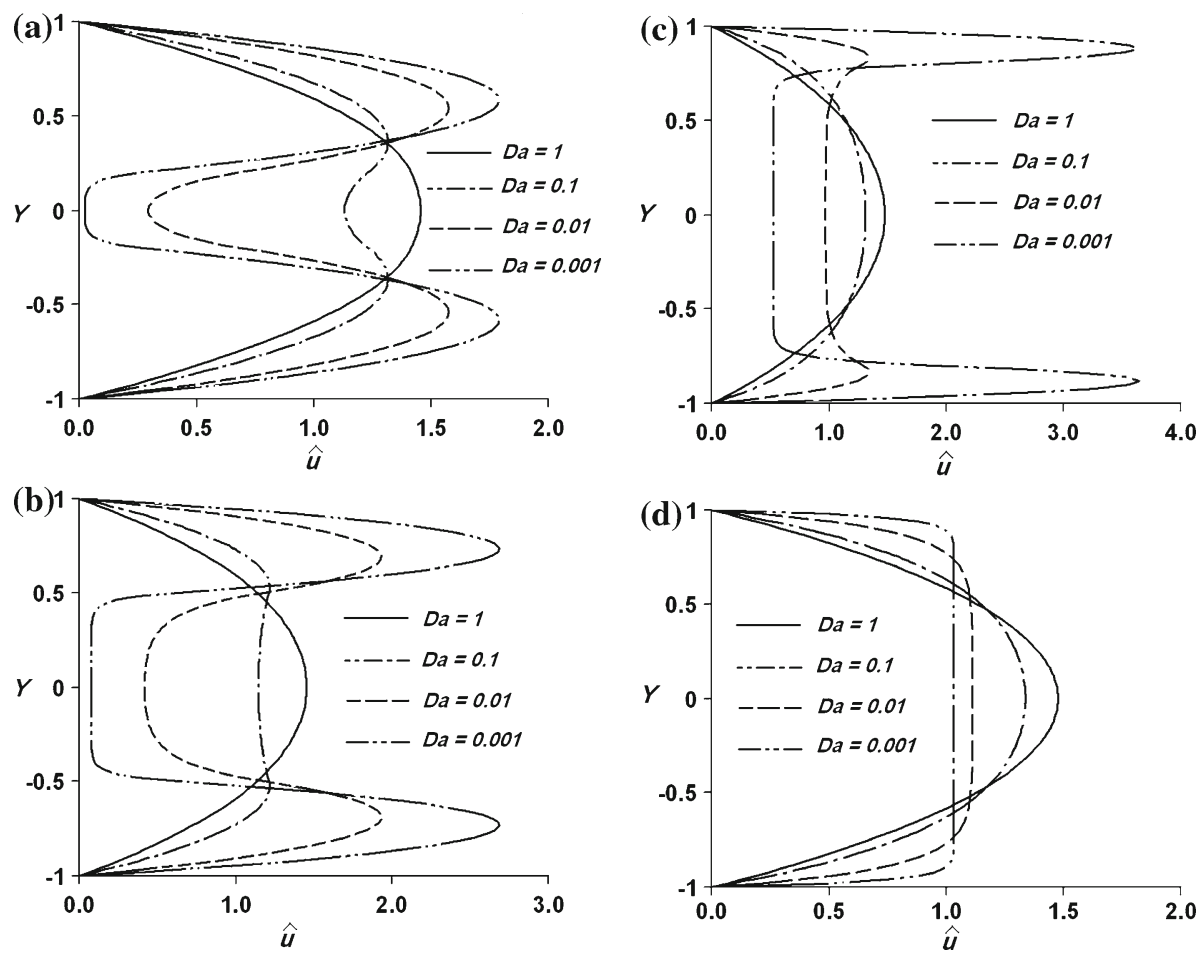

Fig. 4 Normalized velocity profiles for four different Darcy numbers, $\mathbf{a} \xi=0.2, \mathbf{b} \xi=0.5, \mathrm{c} \xi=0.8$, and d $\xi=1$

\subsection{Velocity Profile}

Figure 4 shows the dimensionless normalized velocity profile for four different Darcy number $(D a=1,0.1,0.01$, and 0.001$)$ and four different porous layer thickness $(\xi=0.2,0.5$, 0.8 , and 1 ). Figure 4 a shows the velocity profile for a channel with $\xi=0.2$. The velocity profile of $D a=1$ is identical with the velocity profile of channel with fully clear fluid. By decreasing of Darcy number, the velocity profile is changed. For the low Darcy numbers such as $D a=0.01$ and $D a=0.001$, the effect of porous layer on the velocity profile is clearly seen. The porous layer slows down the fluid velocity at the center of channel while the fluid has high velocity at the upper and lower clear fluid regions of the channel. Figure $4 \mathrm{~b}$ shows the velocity profile for the dimensionless porous layer thickness of $1(\xi=0.5)$. Similar to Fig. 4a, the velocity profile is parabolic and identical with the velocity profile of fully clear fluid channel when $D a=1$. For the low values of Darcy number (i.e., $D a=0.001$ ), the effect of porous layer on the velocity profile is observed. Comparison of Fig. $4 \mathrm{a}$ and $\mathrm{b}$ shows that by increasing porous layer thickness from 0.4 to 1 , the velocity in the center region of channel decreases while the velocity at the upper and lower free regions increases considerably particularly when $D a=0.001$. Figure $4 c$ indicates the velocity profile in the channel when the porous layer thickness is $1.6(\xi=0.8)$. The same parabolic velocity profile for $D a=1$ is observed. The steep velocity gradient at the interface between the porous media and fluid regions indicates the validity of slip boundary condition for flows in channel with low Darcy number (i.e., $D a=0.001$ ). The velocity profile in the channel with fully porous 

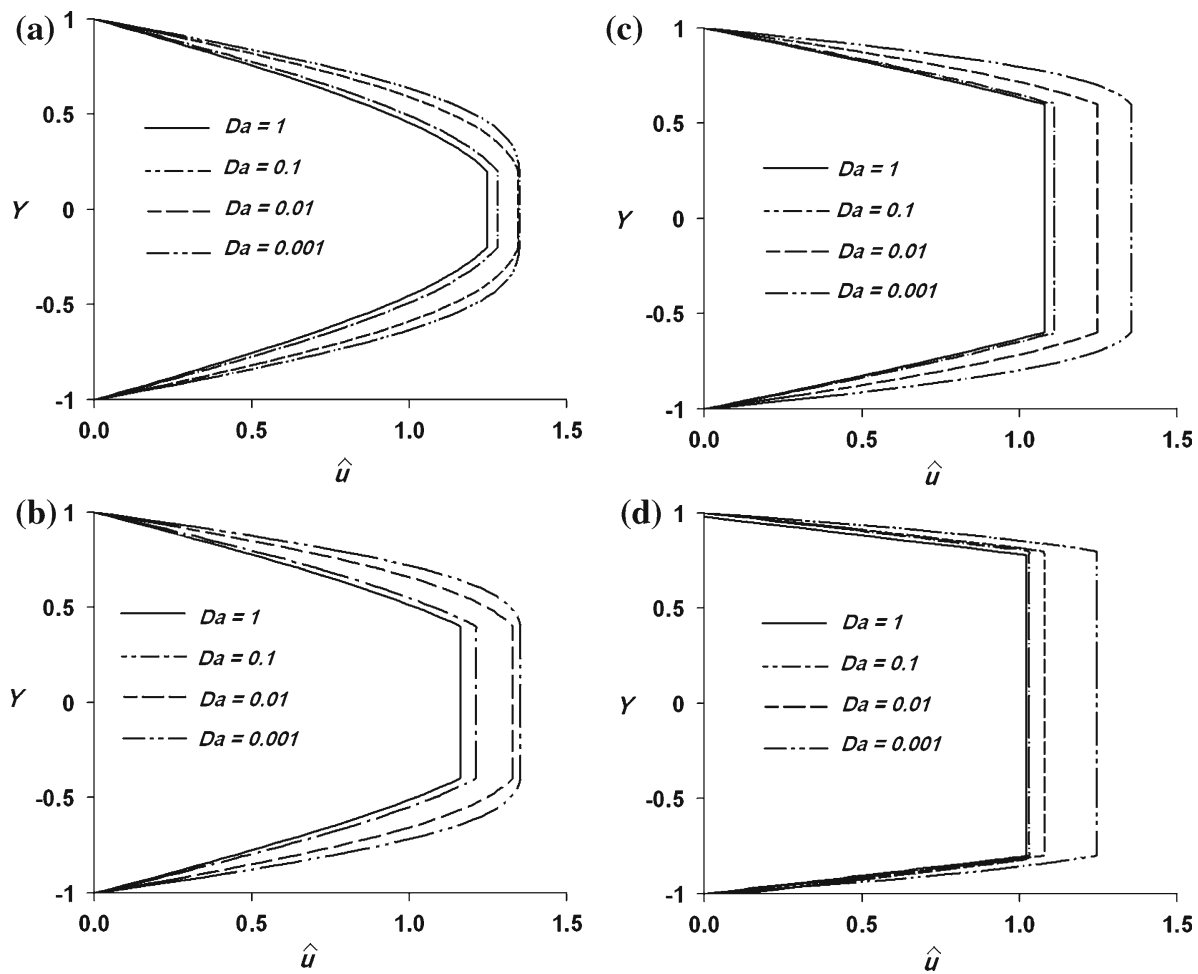

Fig. 5 Dimensionless temperature profiles for four different Darcy numbers when $k_{\mathrm{r}}=10^{4}$, a $\xi=0.2$, b $\xi=0.4, \mathbf{c} \xi=0.6$, and $\mathbf{d} \xi=0.8$

media is shown in Fig. 4 d. The velocity profile for $D a=1$ is identical with other partially porous filled channels of Fig. 4. The flatten velocity profile in the most region of channel for $D a=0.001$ refers to the mixing of fluid due to obstacles in the porous medium.

\subsection{Temperature Profile}

Figure 5 shows the change of temperature profile with Darcy number and porous layer thickness for thermal conductivity ratio of 10,000. Figure 5a shows the dimensionless temperature profile for porous layer thickness of 0.4 . As seen, a uniform temperature distribution is observed in the porous layer due to high thermal conductivity ratio. By increasing Darcy number, the temperature gradient at the wall surface increases refers to increase of Nusselt number. The same changes in dimensionless temperature profiles can be observed for the different porous layer thickness of 0.8 (Fig. 5b), 1.2 (Fig. 5c), and 1.8 (Fig. 5d). Figure 6 shows the dimensionless temperature profiles for different thermal conductivity ratio from 0.1 to 10,000 when $D a=10^{-3}$ and porous layer thickness is 1.8 . For $k_{\mathrm{r}}=0.1$, the gradient of temperature at the wall is not high, if it is compared with the temperature gradient of other thermal conductivity ratios. Hence, a relatively low Nusselt number is expected for a channel with $k_{\mathrm{r}}=0.1$. For $k_{\mathrm{r}}=1$, the gradient of temperature at the interface between the clear fluid and porous layer are the same, that is why a parabolic temperature profile is seen. By increasing thermal conductivity ratio from 1 to 100, the temperature gradient at up and lower sides of the interface (clear fluid and porous regions) becomes different as seen from 
Fig. 6 Temperature profiles in the channel with different thermal conductivity ratio when $\xi=0.9$

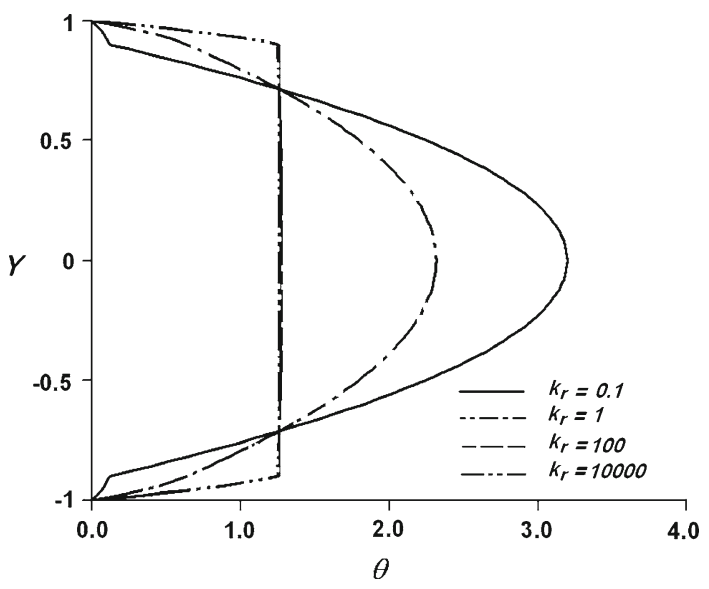

Fig. 6, and uniform temperature distribution is observed in the porous region due to high thermal conductivity of the porous material. The temperature gradient at the channel wall also increases when thermal conductivity ratio is increased from 1 to 100, implying increase of Nusselt number. The temperature profiles are identical for both thermal conductivity ratios of 100 and 10,000 indicating the same Nusselt number for $k_{\mathrm{r}}=100$ and 10,000 .

\subsection{Heat Transfer and Pressure Drop Analysis}

In Fig. 7, the changes of heat transfer increment ratio with porous layer thicknesses are plotted for four different thermal conductivity ratios and Darcy numbers. It should be mentioned that the variation of Nusselt number with $\xi$ is also the same with what shown in Fig. 7. One can calculate Nusselt number from Fig. 7 by multiplication of heat transfer increment ratio (i.e., $\varepsilon_{\text {th }}$ ) with 4.12 which is the Nusselt number of fully clear fluid channel. As it was mentioned before, the heat transfer increment ratio shows the enhancement of heat transfer in a completely clear fluid channel by inserting a porous layer. The change of $\varepsilon_{\text {th }}$ with $\xi$ for $k_{\mathrm{r}}=0.1$ is shown in Fig. 7a. For all Darcy numbers of $10^{-3}, 10^{-4}$, and $10^{-5}$, the heat transfer increment ratio increases with the porous layer thickness and takes a maximum value. After the maximum value, $\varepsilon_{\text {th }}$ drops with further increase in the porous layer thickness. The porous layer thickness for achieving maximum value of $\varepsilon_{\text {th }}$ depends on Darcy number. By inserting a low Darcy number porous layer (such as $D a=0.01$ ), the maximum $\varepsilon_{\text {th }}$ is obtained when the dimensionless porous layer thickness is around 0.4. For porous layer with $D a=10^{-4}$, the maximum of $\varepsilon_{\text {th }}$ can be achieved when $\xi$ is around 0.75 . Figure 7 a also shows that the value of heat transfer increment ratio for a channel can be increased by reducing Darcy number of the inserted porous layer. The parameters of Fig. $7 \mathrm{~b}$ is the same with Fig. 7a, except the thermal conductivity ratio which is $k_{\mathrm{r}}=1$. The heat transfer enhancement ratio is increased by the increase of thermal conductivity ratio; however, the same variation of $\varepsilon_{\text {th }}$ with $\xi$ of Fig. 7a is seen for Fig. 7b. The changes of $\varepsilon_{\text {th }}$ with $\xi$ for $k_{\mathrm{r}}=100$ and 10,000 are shown in Fig. 7c, d, respectively. The maximum heat transfer increment ratio is seen for the channel with fully filled porous media. The comparison of Fig. 7c and d reveals that the increase of thermal conductivity ratio from 100 to 10,000 does not increase the value of $\varepsilon_{\text {th }}$ for $\xi<1$.

Figure 8 shows the changes of $\varepsilon_{\text {th }}$ with $k_{\mathrm{r}}$ for different Darcy number when the porous layer thickness is 1.8. $(\xi=0.9)$. The heat transfer enhancement ratio in the channel is higher for the porous layers with lower Darcy number (i.e., $D a=10^{-5}$ ). The heat transfer 

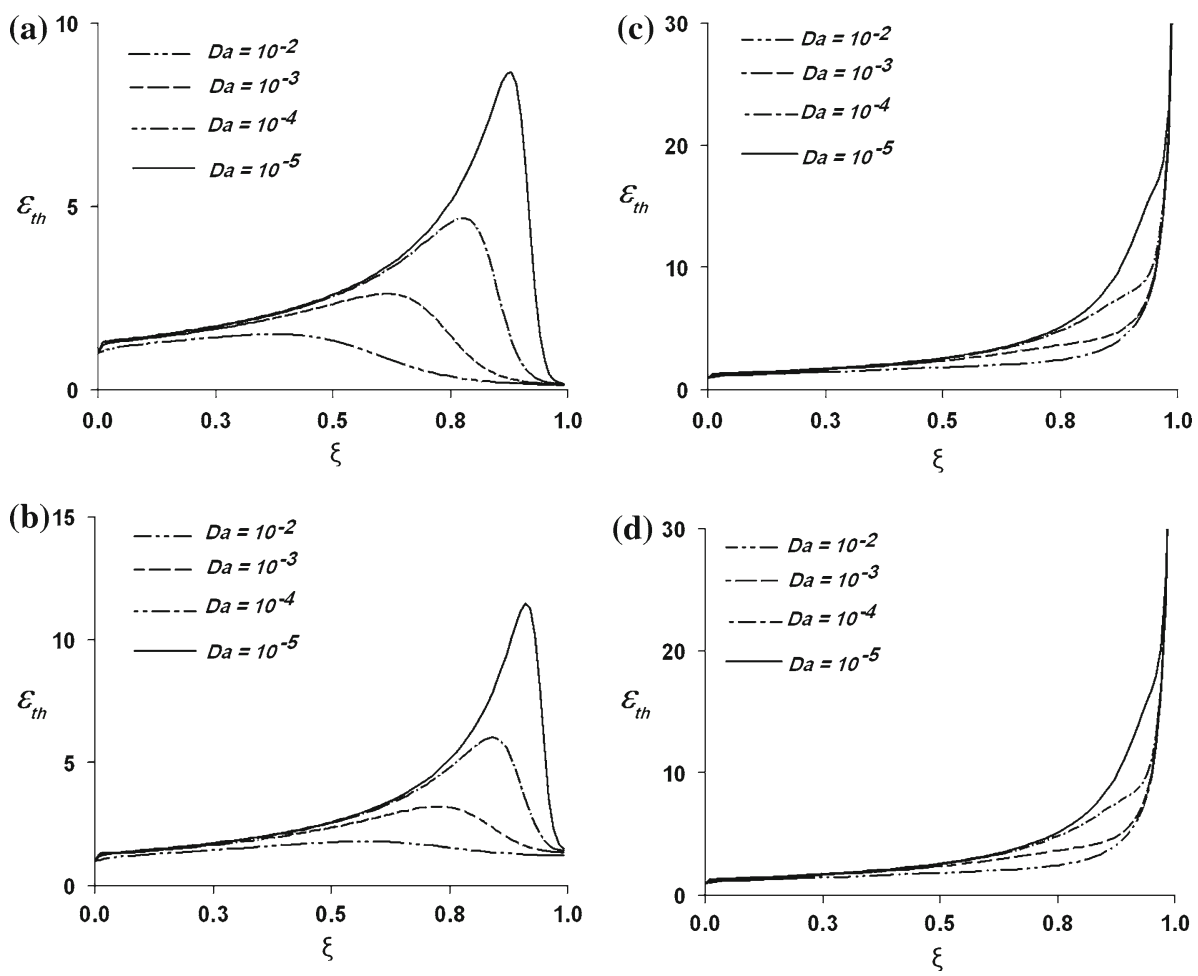

Fig. 7 The variation of heat transfer enhancement ratio with thickness of porous layer for four different Darcy numbers, a $k_{\mathrm{r}}=0.1, \mathbf{b} k_{\mathrm{r}}=1, \mathbf{c} k_{\mathrm{r}}=100, \mathbf{d} k_{\mathrm{r}}=10,000$

Fig. 8 The changes in heat transfer increment ratio with the conductivity ratio for four different Darcy numbers when $\xi=0.9$

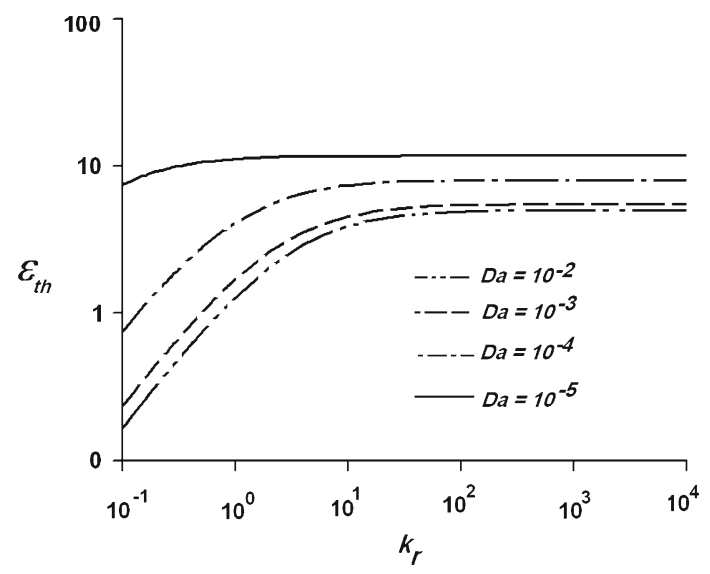

increment ratio is low for small values of $k_{\mathrm{r}}$. It increases with increase of $k_{\mathrm{r}}$ value, however, after a specified value of $k_{\mathrm{r}}$, no heat transfer enhancement is observed. Thus, a suggestion such that higher thermal conductivity ratio provides more heat transfer enhancement may not be correct for $\xi<1$. Figure 8 also shows that the use of porous layer with $D a=10^{-5}$ and $\xi=1.8$ can enhance heat transfer in a channel approximately by 11.8 times. 
Fig. 9 The variation of pressure drop increment ratio with porous layer thickness for four different Darcy numbers

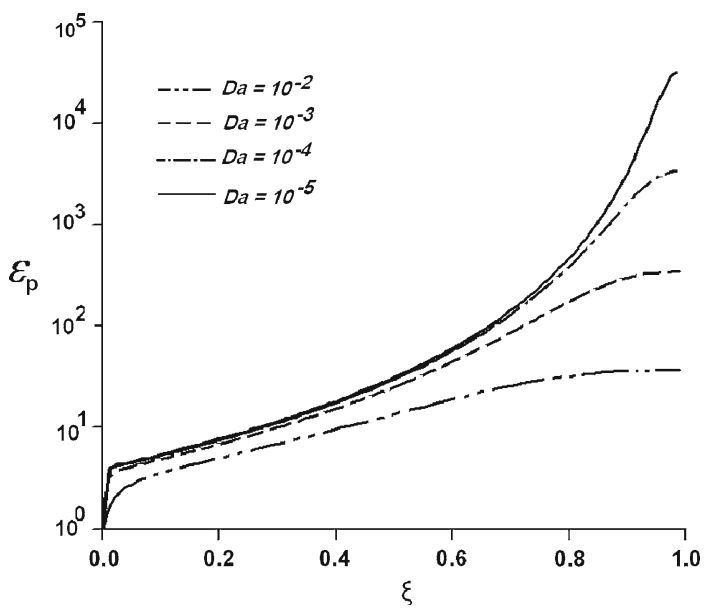

It should be mentioned that for a channel fully filled with porous medium, the Nusselt number proportionally increases with thermal conductivity ratio. For example, the Nusselt number (i.e., $N u_{\mathrm{fp}}$ ) for a channel fully filled with porous medium when $D a=10^{-5}$ and $k_{\mathrm{r}}=1$ is 5.9607 and it increases to $N u_{\mathrm{fp}}=59,607$ for $k_{\mathrm{r}}=10,000$. High thermal conductance of saturated porous medium which is in contact with wall considerably increases heat transfer from the wall to the flowing fluid. For the mentioned case, the value of $\varepsilon_{\text {th }}=14,467$. A similar study was performed by Chang and Lees (2010) for the forced convection in pinfinned channels. It is shown that the heat transfer increment ratio for laminar region is less than 25. Hence, the use of high conductive porous material completely fills the channel might result in higher heat transfer rate compared to the fins and ribs. However, the increase of pressure drop should definitely be taken into account.

The main drawback of the use of porous medium layer, for heat transfer enhancement in a channel or duct is the increase of pressure drop that causes the increase of fan or pump power. Both the heat transfer and pressure drop analysis should be performed to decide on the use of porous layer for heat transfer enhancement. The parameter of $f R e$ is an appropriate indicator to compare the pressure drop of different channels with different values of Darcy number and porous layer thickness. The pressure drop increment ratio $\left(\varepsilon_{\mathrm{p}}\right)$ indicates the increase of pressure drop due to an inserted porous layer in a completely channel. In this problem, $\varepsilon_{\mathrm{p}}$ is the function of Darcy and $\xi$. Figure 9 shows the variation of $\varepsilon_{\mathrm{p}}$ with porous layer thickness for different values of Darcy number. It should be mentioned that the multiplication of $\varepsilon_{\mathrm{p}}$ and 24 yields the channel friction coefficient, as the friction coefficient of a clear fluid channel is 24 [i.e., $(f R e)_{\mathrm{cl}}=24$ ]. As seen from Fig. 9, the pressure drop increment ratio considerably increases with increase of porous layer thickness. It also increases with the decrease of Darcy number. When Darcy number is small, a considerable increment in pressure gradient can be observed even for a very thin porous layer. For instance, for a very thin porous layer of $\xi=0.05$ and $D a=10^{-4}$, the value of $\varepsilon_{\mathrm{p}}$ is 4.5 . This means that the pressure drop in the channel increases by 4.5 times even a thin porous layer is inserted in the channel. For the region of $\xi<0.6$, the pressure drop increment ratio increases with constant rate. However, for the region with $\xi>0.6$, the pressure drop increment ratio is considerably increased particularly for low Darcy numbers (i.e., $D a=10^{-5}$ ). For a channel with $D a=10^{-5}$, the increase of porous layer thickness from 0.2 to 0.3 causes the increase of $\varepsilon_{\mathrm{p}}$ from 7 to 11 . For the same channel, the increase of the porous layer thickness from 0.8 to 0.9 increases $\varepsilon_{\mathrm{p}}$ 

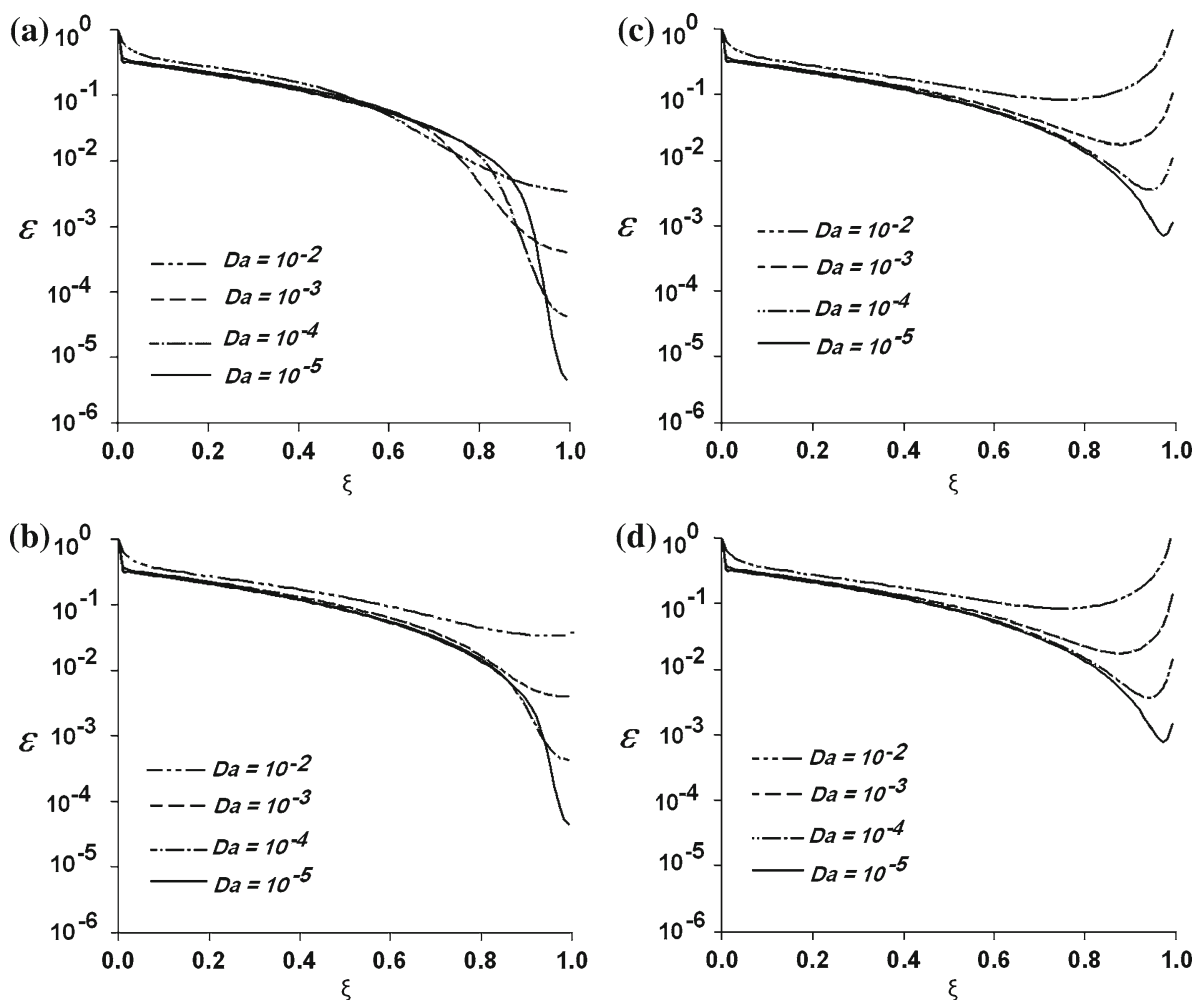

Fig. 10 The changes in overall performance with porous layer thickness for four different Darcy numbers, a $k_{\mathrm{r}}=0.1, \mathbf{b} k_{\mathrm{r}}=1, \mathbf{c} k_{\mathrm{r}}=100, \mathbf{d} k_{\mathrm{r}}=10,000$

from 460 to 3,180. It should be mentioned that the increase of porous layer thickness also increases velocity in the lower and upper clear fluid regions of channel, and that is why the pressure drop increases, considerably.

To have an overall idea on evaluation of an inserted porous layer in a channel both from heat transfer and pressure drop points of view, Fig. 10 is presented. The variation of overall performance, $\varepsilon=\varepsilon_{\mathrm{th}} / \varepsilon_{\mathrm{p}}$, versus porous layer thickness is plotted for different values of Darcy number and thermal conductivity ratio. Figure 10a shows variation of $\varepsilon$ with $\xi$ for $k_{\mathrm{r}}=0.1$. The value of $\varepsilon$ for the region close to $\xi=0$, representing almost clear channel, is approximately around 1. By increasing of porous layer thickness, the value of $\varepsilon$ decreases and becomes less than 1 , showing higher increment of pressure drop in the channel compared to the heat transfer enhancement. For the porous layer thickness of $\xi<0.75$, the values of overall performance for different Darcy numbers are almost the same. For the porous layer thickness between 0.75 and 0.85 , it is observed that the porous layer with $D a=10^{-5}$ provides higher overall performance due to higher heat transfer increment ratio as seen from Fig. 7a. For the porous layer thickness of $\xi>0.9$, the overall performance of $D a=10^{-5}$ rapidly drops due to the rapid decrease of $\varepsilon_{\text {th }}$ (Fig. 7a). By increasing of thermal conductivity ratio from 0.1 to 1 , the variation of overall performance with $\xi$ slightly changes. The channel with porous layer of $D a=10^{-2}$ has maximum overall performance while the minimum overall performance belongs to the channel with $\mathrm{Da}=10^{-5}$ whose pressure drop is the highest (Fig. 10b). It should be noted that the overall performance of $k_{\mathrm{r}}=1$ is considerably 
Fig. 11 The changes in overall performance with thermal conductivity ratio for four different Darcy numbers when $\xi=0.9$

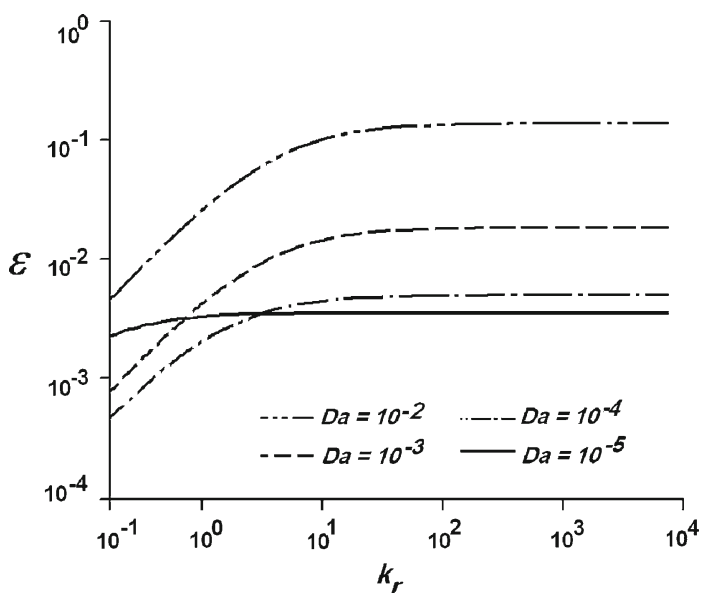

greater than $k_{\mathrm{r}}=0.1$ due to higher enhancement of heat transfer. The variation of $\varepsilon$ with $\xi$ for $k_{\mathrm{r}}=100$ is different than that of $k_{\mathrm{r}}=0.1$ and 1 . The overall performance decreases with increase of porous layer thickness up to a specified porous layer thickness. After a porous layer thickness, the overall performance increases with $\xi$ as the high conductive porous layer become very close to the channel walls. The comparison of Figs. 10b, c shows that the overall performance considerably increases with increase of thermal conductivity ratio from 1 to 100 . Figure $10 \mathrm{~d}$ shows the variation of $\varepsilon$ with $\xi$ for $k_{\mathrm{r}}=10,000$. This figure is almost identical with Fig. 10c. The increase of thermal conductivity ratio from 100 to 10,000 does not result in increasing overall performance. Figure 10c, d, for which $k_{\mathrm{r}}=100$ and 10,000, also shows that the maximum overall performance occurs when the channel is completely filled with porous material. The overall performance of channels fully filled with porous medium highly depends on thermal conductivity ratio. For instance, the thermal performance of a channel fully filled with porous material of $k_{\mathrm{r}}=0.1$ and $D a=10^{-3}$ is 0.0004 indicating higher increase of pressure drop compared to the heat transfer enhancement. By increasing thermal conductivity ratio from 0.1 to 10,000 , the overall performance become 40.31 showing higher heat transfer enhancement compared to increase of pressure drop.

Figure 11 shows the changes in overall performance with thermal conductivity ratio for a channel with porous layer of $\xi=0.9$. Three remarks can be concluded from this figure. First, the overall performance does not change with thermal conductivity ratios for the range of $k_{\mathrm{r}}>10$. Second, for the region of thermal conductivity ratio of $k_{\mathrm{r}}>10$, the decrease of Darcy number decreases the overall performance. For this region, the channel with porous layer of $D a=10^{-2}$ has the highest overall performance while the minimum overall performance is observed for the channel with $D a=10^{-5}$. Finally, for the thermal conductivity ratio of $k_{\mathrm{r}}<0.5$, the overall performance of porous layer with $D a=10^{-5}$ is greater than $D a=10^{-3}$ and $10^{-4}$ due to high heat transfer increment ratio of $D a=10^{-5}$ as seen from Fig. 7a.

\section{Conclusions}

In this study, a laminar, hydrodynamically, and thermally fully developed flow in a channel partially filled with porous medium under symmetrical boundary heat flux condition is 
investigated. Three different parameters are defined to decide on the use of a porous layer in the core of the channel for enhancement of heat transfer. Heat transfer increment ratio is defined to find out the heat transfer enhancement by using an inserted porous layer. Pressure drop increment ratio is defined to determine the increment rate of pressure drop if a porous layer is located at the center of channel. Finally, the overall performance is used to evaluate the use of porous layer from both heat transfer enhancement and pressure drop increment points of view. Based on the obtained results, the following remarks can be concluded:

- Heat transfer increment ratio increases with decrease of Darcy number of porous layer. For $k_{\mathrm{r}}=0.1$ and 1 , there is a porous layer thickness for which maximum enhancement of heat transfer occurs. For $k_{\mathrm{r}}=100$ and 10,000, maximum heat transfer enhancement is achieved for a porous layer completely fills a channel.

- For low values of thermal conductivity ratio $\left(k_{\mathrm{r}}<100\right)$, the increase of $k_{\mathrm{r}}$ increases heat transfer enhancement ratio. However, for high values of thermal conductivity $\left(k_{\mathrm{r}}>100\right)$, heat transfer enhancement ratio is not highly influenced from $k_{\mathrm{r}}$.

- The decrease in Darcy number causes increase of pressure drop in the channel. High values of pressure drop increment ratio is observed for porous layer thickness of $\xi>0.8$ particularly when $D a=10^{-5}$.

- For the channels partially filled porous medium, the value of overall performance is less that unity indicating that the increase of pressure drop is greater than heat transfer enhancement.

- For the channel completely filled with porous material having high thermal conductivity ratio $\left(k_{\mathrm{r}}>100\right)$ and high values of Darcy numbers $\left(D a>10^{-3}\right)$, the value of overall performance is greater than unity as high conductive porous material is in contact with channel walls.

A detailed study should be performed on the comparison of heat transfer and pressure drop increment ratios between the channels assisted with fins and porous materials under the same conditions. This study can be performed for other positions of porous layer in a channel to find out the effects of the layer position on the overall performances.

Acknowledgments The authors wish to express their sincere thanks to the reviewers for their valuable comments and suggestions.

\section{References}

Alazmi, B., Vafai, K.: Analysis of fluid flow and heat transfer interfacial conditions between a porous medium and a fluid layer. Int. J. Heat Mass Transf. 44, 1735-1749 (2001)

Alkam, M.K., Al-Nimr, M.A.: Transient non-Darcian forced convection flow in a pipe partially filled with a porous material. J. Heat Mass Transf. 41, 347-356 (1998)

Al-Nimr, M.A., Alkam, M.K.: A modified tubeless solar collector partially filled with porous substrate. Renew. Energy 13, 165-173 (1998)

Bear, J.: Dynamics of fluid in porous media. Elsevier, New York (1972)

Bejan, A., Dincer, I., Lorente, S., Miguel, A.F., Reis, A.F.: Porous and complex flow structures in modern technologies. Springer, New York (2004)

Calmidi, V.V., Mahajan, R.L.: Forced convection in high porosity metal foams. J. Heat Transf. 122:557-565 (2000)

Chang, S.W., Lees, A.W.: Endwall heat transfer and pressure drop in scale-roughened pin-fin channels. Int. J. Therm. Sci. 49, 702-713 (2010)

Chikh, S., Boumedien, A., Bouhadef, K., Lauriat, G.: Analytical solution of non-darcian forced convection in an annular duct partially filled with a porous medium. Int. J. Heat Mass Transf. 38:1543-1551 (1995)

Forooghi, P., Abkar, M., Saffar-Avval, M.: Steady and unsteady heat transfer in a channel partially filled with porous media under thermal non-equilibrium condition. Transp. Porous Media 86, 177-198 (2011) 
Ingham, D.B., Pop, I. (eds.): Transport phenomena in porous media. Elsevier, Oxford (2005)

Kuznetsov, A.V.: Analytical investigation of the fluid flow in the interface region between a porous medium and a clear fluid in channels partially filled with a porous medium. Appl. Sci. Res. 56, 53-67 (1996)

Kuznetsov, A.V.: Influence of the stress jump boundary condition at the porous medium clear-fluid interface on a flow at a porous wall. Int. Commun. Heat Mass Transf. 24, 401-410 (1997)

Kuznetsov, A.V.: Analytical investigation of Couette flow in a composite channel partially filled with a porous medium and partially with a clear fluid. Int. J. Heat Mass Transf. 41, 2556-2560 (1998)

Kuznetsov, A.V.: Forced convective heat transfer in a parallel-plate channel with a porous core. Int. J. Appl. Mech. Eng. 4, 271-290 (1999)

Kuznetsov, A.V.: Analytical studies of forced convection in partly porous configurations. In: Vafai, K. (ed.) Handbook of porous media, pp. 269-312. Marcel Dekker, New York (2000)

Kuznetsov, A.V., Nield, D.A.: Forced convection in a channel partly occupied by a bidisperse porous medium: asymmetric case. Int. J. Heat Mass Transf. 53, 5167-5175 (2010)

Morosuk, T.V.: Entropy generation in conduits filled with porous medium totally and partially. Int. J. Heat Mass Transf. 48, 2548-2560 (2005)

Najjari, M., Nasrallah, S.B.: Effects of latent heat storage on heat transfer in a forced flow in a porous layer. Int. J. Therm. Sci. 47, 825-833 (2008)

Nakayama, A., Kuwahara, F., Sano, Y.: Concept of equivalent diameter for heat and fluid flow in porous media. AICHE 53, 732-736 (2007)

Nield, D.A.: The Beavers-Joseph boundary condition and related matters: a historical and critical note. Transp. Porous Media 78, 537-540 (2009)

Nield, D.A., Bejan, A.: Convection in porous media (3rd edn). Springer, New York (2006)

Nield, D.A., Kuznetsov, A.V.: Forced convection in a channel partly occupied by a bidisperse porous medium: symmetric case. J. Heat Transf. 133, 072601 (2011)

Ould-Amer, Y., Chikh, S., Bouhadef, K., Lauriat, G.: Forced convection cooling enhancement by use of porous materials. Int. J. Heat Fluid Flow 19, 251-258 (1998)

Pop, I., Ingham, D.B.: Convective heat transfer: mathematical and computational modeling of viscous fluids and porous media. Pergamon, Oxford (2001)

Poulikakos, D., Kazmierczak, M.: Forced convection in a duct partially filled with a porous material. ASME J. Heat Transf. 109, 653-662 (1987)

Satyamurty, V.V., Bhargavi, D.: Forced convection in thermally developing region of a channel partially filled with a porous material and optimal porous fraction. Int. J. Therm. Sci. 49, 319-332 (2010)

Shokouhmand, H., Jam, F., Salimpour, M.R.: The effect of porous insert position on the enhanced heat transfer in partially filled channels. Int. Commun. Heat Mass Transf. 38, 1162-1167 (2011)

Vadasz, P. (ed.): Emerging topics in heat and mass transfer in porous media. Springer, New York (2008)

Vafai, K.: Handbook of porous media. Taylor \& Francis, New York (2005)

Vafai, K.: Porous media: applications in biological systems and biotechnology. CRC Press, Tokyo (2010)

Vafai, K., Kim, S.J.: Fluid mechanics of the interface region between a porous medium and a fluid layer-an exact solution. Int. J. Heat Mass Transf. 11, 254-256 (1990)

Yang, C., Liu, W., Nakayama, A.: Forced convective heat transfer enhancement in a tube with its core partially filled with a porous medium. Open Transp. Phenom. J. 1, 1-6 (2009) 